\title{
Portfolio Research Based on Differential Evolution Method
}

\author{
Yulian Fan, Yang $\mathrm{Xu}^{*}$ and Mengying Chang \\ College of Science, North China University of Technology, 100144, Beijing, China \\ ${ }^{*}$ Corresponding author
}

\begin{abstract}
Portfolio optimization refers to the fact that investors allocate funds to certain kinds of assets, so that the investment amount of each type of assets accounts for a certain proportion of the total investment. The purpose is to make the overall income of the assets held by investors as high as possible, or make the investment risk as low as possible. With the deepening of people's research, the theory of portfolio optimization has been gradually applied into a more successful and more mature theory. This paper intends to use the basic prospect theory model, using Matlab software, based on the differential evolution algorithm, to optimize the real economic market data of 10 assets.
\end{abstract}

Keywords-portfolio optimization; prospect theory model; differential evolution algorithm

\section{FOREWORD (STUDY BACKGROUND)}

With the continuous development of China's economy and the deepening of economic system reform, the concept of financial management of Chinese nationals has gradually improved, and investment has gradually become a widely used investment channel. Investing can make us profitable, but investors also have to bear certain risks while gaining income. How can investors reasonably allocate funds to invest in different assets, determine the appropriate proportion of investment in a variety of assets to the total amount of investment, so that the total income of the assets held by investors is as high as possible and the risk is as low as possible. How to calculate the risks and benefits of portfolio investment and how to allocate assets to achieve a certain balance between these two indicators. These are all issues that investors need to solve urgently. In this research context, this paper has conducted in-depth research on portfolio optimization.

\section{Portfolio Optimization Problem}

\section{A. Introduction}

The main purpose of a good stock investment is to effectively avoid stock market risks and get the most benefit. Modern asset portfolio theory was first developed by American economist Harry M. Markowit (1927) proposed, he applied the mean and variance of portfolio compensation and found the optimal solution by establishing a mathematical model. The expected utility was first proposed by the Swiss mathematician Bemoulli, and by VonNeumann, Morgenstem et al., based on the answer to the "St. Petersburg Paradox" by Bemoulli, after strict axiomatization assumptions. It describes how "rational people" determine optimal decisions in the face of uncertain situations. The theory of prospects is based on the expected utility, adding important psychological components and considering human behavior in the decision-making process. This paper will use the prospect theory model to calculate the optimal solution of the model through differential evolution algorithm.

\section{B. Symbol hypothesis}

Assuming $N$ is the number of assets and $\omega_{i} \geq 0$ is the weight of asset $i$ in the portfolio, then a portfolio can be expressed as $x_{i}=\left(\omega_{1}, \cdots, \omega_{N}\right), \sum_{i=1}^{N} \omega_{i} . S$ is assumed to be the number of situations, $p_{s}$ is the probability of occurrence of $s$ moment, $r_{i s}$ is the return of asset $i$ at time $s, \bar{r}_{i}$ is the average return of asset $i$, and $d$ is the ideal return level.

\section{BASIC PROSPECT TheORY MODEL}

The prospect theory is a decisive paradigm decision model proposed by Kahneman and Tversky (1979).It divides risk decisions into two processes: editing and evaluation. In the editing phase, the individual's gains and losses in the portfolio are determined by defining a reference point $r_{0}$, where $r_{0}$ represents the zero gain or zero loss that the individual believes. In the evaluation phase, the weight function $\pi(p)$ and the value function $v(r)$ that depend on the subjective probability are used to judge the information.

In the prospect theory, the probability weight function $\pi(p)$ is to measure the effect of the event on the desirability of the prospect. $\pi(p)$ is an increasing function, $\pi(0)=0, \pi(1)=1$, when the probability $p$ is very small, then $\pi(p) \geq p$.

In the prospect theory, the value function $v(r)$ is the behavior value that describes the result of the gain or loss. For a given reference point $r_{0}$, the image is usually asymmetrical because the function reflects the attitude of different investors towards gains and losses; for the benefit is upward concave, for the loss is convex downward. Furthermore, typically the value function $v(r)$ is steeper for the loss than for the gain, for $s>0$, there is $v\left(r_{s}\right) \leq-v\left(r_{-s}\right)$. In this paper, the value function uses the explicit formula given in Tversky and Kahneman (1992):

$$
v(r)=\left\{\begin{array}{c}
\left(r-r_{0}\right)^{\alpha}, \text { if } r \geq r_{0}, \\
-\lambda\left(r_{0}-r\right)^{\beta} \text {,if } r \leq r_{0},
\end{array}\right.
$$


Among them, $\alpha=\beta=0.88$ represents the corresponding risk and loss risk aversion coefficient, loss aversion coefficient $\lambda=2.25$. To simplify the study, the weight function $\pi(p)=p$. Therefore, the prospect theoretical utility function can be represented by $\pi(p)$ and $v(r)$ :

$$
\mathrm{PT}_{\mathrm{U}}=\sum_{s=1}^{S} \pi\left(p_{s}\right) v\left(r_{s}\right)=\sum_{s=1}^{S} p_{s} v\left(\sum_{i=1}^{N} r_{s i} \omega_{i}\right) .
$$

According to the prospect theory, if the optimal portfolio is to be found, the prospect theoretical utility function is required to be maximized. The basic prospect theoretical model can be expressed as:

$$
\begin{gathered}
\operatorname{maxPT}(\mathrm{x})=\sum_{s=1}^{S} p_{s} v\left(\sum_{i=1}^{N} r_{s i} \omega_{i}\right) \\
\left\{\begin{array}{l}
\bar{r}(x)=\sum_{i=1}^{N} \bar{r}_{i} \omega_{i} \geq d \\
\sum_{i=1}^{N} \omega_{i}=1, \\
\omega_{i} \geq 0, i=1, \cdots, N .
\end{array}\right.
\end{gathered}
$$

\section{DifFERENTIAL EVOLUTION ALGORITHM}

This paper uses differential evolution algorithm to solve the above portfolio optimization problem. The Differential Evolution (DE) was first proposed by Storn and Price in 1995.It is a heuristic method like the genetic algorithm (GA), and it is an optimization algorithm based on modern intelligence theory. The main idea of the algorithm is to randomly generate an initial population, and through evolution, crossover and selection, continuously evolve to guide the search to the optimal solution.

\section{A. Basic steps of differential evolution algorithm}

1) Population initialization. Assuming $\mathrm{N}$ is the number of available assets, randomly generate $P^{2}$ individuals, each consisting of N-dimensional vectors: $x_{i}=\left(\omega_{1}, \cdots, \omega_{N}\right), \forall i=1, \cdots, P^{2}$.

2) Variation.In each iteration, randomly select 3 individuals, $x_{a}, x_{b}, x_{c}$ and guarantee $x_{a} \neq x_{b} \neq x_{c} \neq x_{i}$, generate a variation vector: $h_{i}=x_{a}+F \cdot\left(x_{b}-x_{c}\right)$. Where $F$ is the scaling factor, $F \in[0,2]$.

3) Cross. Intersect the mutated vector with the conditions of:

$$
\tilde{x}_{i}=\left\{\begin{array}{l}
h_{i}, R=j \text { or } \operatorname{rand}(0,1) \leq C R, \\
x_{i}, \text { else. }
\end{array}\right.
$$

Where $R \in\{1, \cdots, N\}$ is a randomly generated random number and $C R$ is the crossover probability, $C R \in[0,1]$.

4) Select. By calculating $\operatorname{PT}\left(\tilde{x}_{i}\right)$ and $\operatorname{PT}\left(x_{i}\right)$,the largest individual $\tilde{y}_{i}$ is selected as the new individual to enter the next iteration. In the last generation $g=G, \tilde{y}_{i}$ of choice $\max \operatorname{PT}\left(\tilde{y}_{i}\right)$ becomes the optimal solution $y_{i}$.

\section{B. Differential evolution pseudo code}

Here, the pseudo code giving the differential evolution algorithm is for reference only. This code is just a basic framework that needs to be written further based on different objective functions and constraints.



\section{INSTANCE TEST}

In this paper, the program for the above model is written in Matlab. The data uses the 10 constituent stocks with the highest weight in the CSI 300 Index. The daily closing price $p_{i s}$ is selected from August 1, 2018 to August 31, 2018. Here, the logarithm of the price ratio is used as the daily rate of return, $r=\ln \left(\frac{p_{i s}}{p_{i s-1}}\right), i=1, \cdots, N ; s=1, \cdots, S$. Set the scaling factor $F=0.8$, the crossover probability $C R=0.5$, the number of iterations $G$ $=100, P=20$.

The results are as shown in table I. As can be seen from the numerical value, the sum of the weights in the calculated portfolio is approximately 1 . The trend of the PT value obtained by 100 iterations is shown in Figure 1. As can be seen from the upward trend in the figure, the program does find the largest utility value in the iteration. 


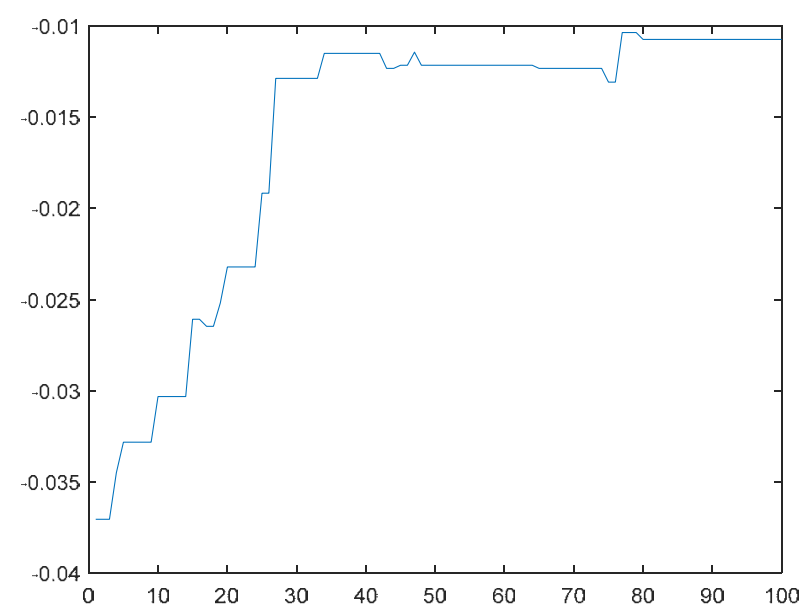

FIGURE I. PT TREND GRAPH OF 100 ITERATIONS

TABLE I. OPTIMAL PORTFOLIO OF TESTS

\begin{tabular}{|c|c|c|c|c|c|c|c|c|c|}
\hline$\omega_{1}$ & $\omega_{2}$ & $\omega_{3}$ & $\omega_{4}$ & $\omega_{5}$ & $\omega_{6}$ & $\omega_{7}$ & $\omega_{8}$ & $\omega_{9}$ & $\omega_{10}$ \\
\hline 0.1782 & 0.0172 & 0.2915 & 0.0227 & 0.0073 & 0.1712 & 0.0232 & 0.2209 & 0.0176 & 0.0555 \\
\hline
\end{tabular}

\section{SUMMARY}

In this paper, the differential evolution algorithm is used to solve the prospect theoretical model, and the feasibility of this method in portfolio research is verified by an example test. The user can change different scaling factors, crossover probabilities, and iteration times according to the actual problem to obtain a better solution.

\section{ACKNOWLEDGMENT}

Project source: North China University of Technology, No. $107051360018 X N 012 / 037$.

\section{REFERENCES}

[1] Markowitz, H., Portfolio selection. J. Finance, 1952, 7, 7-91.

[2] Kahneman, D. and Tversky, A., Prospect theory: An analysis of decision under risk. Econometrica, 1979, 47, 263-291.

[3] Tversky, A. and Kahneman, D., Advances in prospect theory: Cumulative representation of uncertainty. J. Risk Uncertainty, 1992, 5, 297-323.

[4] Akbaripour, H. and Masehian, E., Efficient and robust parameter tuning for heuristic algorithms. Int. J. Ind. Eng. Prod. Res., 2013, 24, 143-150.

[5] Lia,Y. andYang, L., Prospect theory, the disposition effect and asset prices. J. Financial Econ. 2013, 107, 715-739.

[6] Taras Bodnar,Wolfgang Schmid. On the exact distribution of the estimated expected utility portfolio weights: Theory and applications[J]. with Applications in Finance and Insurance,2011,28(4).

[7] Wei-Guo Zhang,Yong-Jun Liu,Wei-Jun Xu. A new fuzzy programming approach for multi-period portfolio optimization with return demand and risk control[J]. Fuzzy Sets and Systems,2014,246. 